I N S T I T U T O

$\mathrm{DE}$

M E D I C I N A

T R O P I C A L

DE

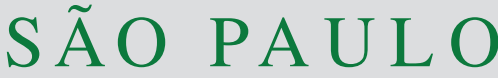

JOURNAL OF THE SÃO PAULO INSTITUTE OF TROPICAL MEDICINE

${ }^{1}$ University of Kragujevac, Faculty of Medical Sciences, Department of Pharmacology and Toxicology, Kragujevac, Serbia

${ }^{2}$ University of Kragujevac, Faculty of Medical Sciences, Kragujevac, Serbia

${ }^{3}$ University of Kragujevac, Faculty of Medical Sciences, Department of Pharmacy, Kragujevac, Serbia

Correspondence to: Milos N. Milosavljevic Faculty of Medical Sciences, Department of Pharmacology and Toxicology, Svetozara Markovica 69, 34000, Kragujevac, Serbia Tel: +3816 69047886

E-mail: milosavljevicmilos91@gmail.com

Received: 26 January 2021

Accepted: 1 March 2021

\section{Antimicrobial treatment of Erysipelatoclostridium ramosum invasive infections: a systematic review}

\author{
Milos N. Milosavljevic ${ }^{(1}$, Marina Kostic ${ }^{1}$, Jasmina Milovanovic' ${ }^{1}$, Radica \\ Zivkovic Zaric ${ }^{1}$, Milorad Stojadinovic ${ }^{(1)}{ }^{2}$, Slobodan M. Jankovic ${ }^{1}$, Srdjan M. \\ Stefanovic ${ }^{3}$
}

\section{ABSTRACT}

The aim of this systematic review was to determine the causal role of Erysipelatoclostridium ramosum in specific invasive infections in humans, and to assess the clinical outcome of antibiotic therapy used to treat them. Several electronic databases were systematically searched for clinical trials, observational studies or individual cases on patients of any age and gender with a systemic inflammatory response syndrome (SIRS) due to E. ramosum isolated from body fluids or tissues in which it is not normally present. Only reports identifying E. ramosum as the only microorganism isolated from a patient with SIRS were included. This systematic review included 15 studies reporting 19 individual cases in which E. ramosum caused invasive infections in various tissues, mainly in immunocompromised patients. E. ramosum was most often isolated by blood cultures and identified by specific biochemical tests. Severe infections caused by E. ramosum were in most cases effectively treated with antibiotics, except in two patients, one of whom died. More than one isolate of E. ramosum exhibited $100 \%$ susceptibility to metronidazole, amoxicillin/clavulanate and piperacillin/tazobactam. On the other hand, individual resistance of this bacterium to penicillin, ciprofloxacin, clindamycin, imipenem and ertapenem was reported. This systematic review confirmed the clinical relevance of E. ramosum as a cause of a number of severe infections mainly in immunocompromised inpatients. Metronidazole and meropenem appear to be the antibiotics of choice that should be used in combination or as monotherapy to treat E. ramosum infections, depending on the type and severity of the infection.

KEYWORDS: Erysipelatoclostridium ramosum. Gram-positive bacillus. Invasive infection. Antibiotic treatment. Systematic review.

\section{INTRODUCTION}

The recently revised genome-based bacterial taxonomy classified Erysipelatoclostridium ramosum (genus Erysipelatoclostridium) into the family Erysipelatoclostridiaceae, along with other strains of the phylogenetic clostridium VIII species ${ }^{1}$. Previously, this Gram-positive, non-mobile, spore-forming anaerobic bacillus was known for a long time as Clostridium ramosum, while it was called Bacillus ramosum and Ramibacterium ramosum during the first several decades since its discovery in $1898^{2}$. Erysipelatoclostridium ramosum (E. ramosum) belongs to the human gut commensal microbiota, though some strains have the ability to produce IgA1 and IgA2 proteases, thus increasing the host susceptibility to opportunistic bacterial invasion by translocation across the intestinal mucosa ${ }^{3}$. This particularly affects children under the age of five and immunocompromised elderly people who, 
although rarely, could develop invasive infections ${ }^{4,5}$. Due to the many difficulties to properly grow $E$. ramosum in culture and perform its identification by routine microbiological techniques, the incidence of both, possible endogenous and exogenous infections caused by E. ramosum is considered underestimated in the real-world clinical practicE. The use of modern diagnostic techniques, such as mass spectrometry, has shown that various anaerobic gram-positive bacteria, which are not considered so common, may cause brain abscesses $^{6}$. Invasive infections such as bacteremia ${ }^{5}$, septic arthritis ${ }^{7}$, septic pseudoarthrosis ${ }^{8}$, osteomyelitis ${ }^{9}$, cerebellar abscess $^{10}$, lung abscess ${ }^{11}$, Fournier's gangrene ${ }^{12}$, gas gangrene $^{13}$, spondylodiscitis $^{14}$, pseudomembranous colitis ${ }^{15}$, septic arteria emboli ${ }^{16}$ among others, might be associated with a high risk of death.

To our best knowledge, no evidence-based guidelines have been proposed to date to support adequate antibiotic therapy for Erysipelatoclostridium ramosum infections. Published literature has mostly reported individual cases suggesting certain antibiotics for the treatment of these infections based on in vitro susceptibility testing $4,5,7,14,17,18$. E. ramosum shows excellent susceptibility to piperacillintazobactam, amoxicillin/clavulanate, ampicillin/sulbactam, imipenem, meropenem, metronidazole, vancomycin and chloramphenicol. Sensitivity to penicillin and cephalosporins is variable, most likely due to the production of $\beta$-lactamases by some E. ramosum strains ${ }^{5,14}$. Similarly, discordant results related to clindamycin sensitivity have been reported ${ }^{5,12}$. There is probably an intrinsic resistance to aminoglycosides, fluoroquinolones, rifampin and most of tetracyclines ${ }^{5,14,19}$. In addition, the doses and duration of antibiotic therapy can also vary significantly. Considering all of the above, there is an unmet clinical need to create some kind of guidance for antibiotic treatment of invasive E. ramosum infections that could help physicians to address this rare but potentially serious problem in clinical practice.

Therefore, the goal of the present systematic review was to determine the causal role of E. ramosum in specific invasive infections in humans and to assess the clinical outcomes of antibiotic therapy used to treat them.

\section{MATERIALS AND METHODS}

Reports from clinical trials, observational studies or individual cases including case reports and case series on patients of any age and gender presenting with systemic inflammatory response syndrome (SIRS) due to E. ramosum isolated from body fluids or tissues in which it is not normally present and identified by one or more of the following diagnostic methods: conventional techniques such as colony morphology, standardized biochemical reactions, gas-liquid chromatography, commercial anaerobe kits, among others, as well as more sophisticated and accurate methods, such as Matrix-assisted laser desorption ionization-time-of-flight mass spectrometry (MALDI-TOF MS) and species-specific polymerase chain reaction (PCR) for E. ramosum ${ }^{19}$, were accepted for inclusion in this systematic review. Most importantly, only reports identifying $E$. ramosum as the only microorganism isolated from a patient with SIRS were included. On the other hand, reports published on the cases of $E$. ramosum infections mentioned in review articles, cases of E. ramosum infections in non-human species and cases with incomplete data were excluded from this review.

The strategy of finding relevant published articles was focused on the search of the following electronic database: Medline (PubMed), Scopus, Ebsco database (Discovery Service), Google Scholar, Cochrane database of published clinical trials - Central (Wiley Online Library), ClinicalTrials gov (U.S. National Library of Medicine), and SCIndex (Serbian Citation Index). The search of these electronic databases covered the last 50 years (from 1970 to May 5, 2020). Six authors (SS, MM, JM, MK, RZZ, and MS) performed the search independently. The researcher MM developed the most comprehensive search of the Medline database: (((“bacillus”[MeSH Terms] OR "bacillus”[All Fields]) AND ramosum[All Fields]) OR (Ramibacterium[All Fields] AND ramosum[All Fields]) OR ((“clostridium”[MeSH Terms] OR "clostridium"[All Fields]) AND ramosum[All Fields]) OR (Erysipelatoclostridium[All Fields] AND ramosum[All Fields])) AND ((invasive[All Fields] AND ("infection"[MeSH Terms] OR "infection"[All Fields] OR "infections”[All Fields])) OR ("bacteraemia"[All Fields] OR "bacteremia"[MeSH Terms] OR "bacteremia"[All Fields]) OR ("arthritis, infectious"[MeSH Terms] OR ("arthritis"[All Fields] AND "infectious"[All Fields]) OR "infectious arthritis"[All Fields] OR ("septic"[All Fields] AND “arthritis”[All Fields]) OR "septic arthritis"[All Fields]) OR (septic[All Fields] AND ("pseudarthrosis"[MeSH Terms] OR "pseudarthrosis"[All Fields] OR "pseudoarthrosis"[All Fields])) OR ("peritonitis"[MeSH Terms] OR "peritonitis"[All Fields]) OR ((“cerebellum”[MeSH Terms] OR "cerebellum"[All Fields] OR "cerebellar"[All Fields]) AND ("abscess"[MeSH Terms] OR "abscess"[All Fields])) OR ("brain abscess"[MeSH Terms] OR ("brain"[All Fields] AND "abscess"[All Fields]) OR "brain abscess"[All Fields]) OR ("lung abscess"[MeSH Terms] OR ("lung”[All Fields] AND "abscess"[All Fields]) OR "lung abscess"[All Fields]) OR ("gas gangrene"[MeSH Terms] OR ("gas”[All Fields] AND "gangrene"[All Fields]) OR "gas gangrene"[All Fields]) OR ("fournier gangrene"[MeSH Terms] OR ("fournier"[All Fields] AND "gangrene"[All Fields]) OR 
"fournier gangrene"[All Fields] OR ("fournier's"[All Fields] AND "gangrene"[All Fields]) OR "fournier's gangrene"[All Fields]) OR (“discitis"[MeSH Terms] OR "discitis"[All Fields] OR "spondylodiscitis"[All Fields]) OR ("anaemia”[All Fields] OR "anemia"[MeSH Terms] OR "anemia"[All Fields]) OR ("osteomyelitis"[MeSH Terms] OR “osteomyelitis"[All Fields]) OR ("sepsis"[MeSH Terms] OR "sepsis"[All Fields]) OR ("enterocolitis, pseudomembranous"[MeSH Terms] OR ("enterocolitis"[All Fields] AND "pseudomembranous"[All Fields]) OR "pseudomembranous enterocolitis"[All Fields] OR ("pseudomembranous"[All Fields] AND "colitis"[All Fields]) OR "pseudomembranous colitis"[All Fields])). No restrictions were made regarding the format, language or date of publication. The "snowball" method was also used to search for additional similar articles using references and key words of the retrieved papers.

Initially, the eligibility of retrieved studies was screened based on titles and abstracts, by six authors (SS, MM, JM, MK, RZZ, and MS) independently. In cases in which it was not possible to assess whether the study fully corresponded to the research topic on the basis of abstracts and titles, the full-length article was retrieved and analyzed. Articles were included in the review if all authors (SS, MM, SJ, JM, MK, RZZ and MS) agreed that eligibility criteria were met. If the reviewers had different opinions on the suitability of the study for inclusion, the matter was resolved by the senior author (SJ).

The data extracted from the included papers were entered in Excel table and assessed for: (1) Publication ID, (2) Report ID, (3) Review author initials, (4) Citation and contact details, (5) Eligibility for review, (6) Study design, (7) Total study duration, (8) Risk of bias, if applicable (randomization if any, sequence generation, allocation sequence concealment, blinding, unequal loss of participants from a study, and other concerns about bias), (9) Total number of patients, (10) Age of patients, (11) Gender of patients, (12) Setting, (13) Country, (14) Presence of comorbidities or underlying immunocompromising acute or chronic conditions, such as prior antibiotic use and/or surgery or traumatic injury and/or diabetes mellitus and/or cancer and/or renal or liver failure, among others, (15) Site of isolation of the bacterium (body fluid or tissue), (16) Sampling method, (17) Method of E. ramosum identification (biochemical methods and/ or MALDI TOF and/or 16S RNA sequencing or other routine microbiological techniques for detecting specific anaerobes, if used), (18) Maximal level of C-reactive protein (CRP) during the infection, (19) Maximal level of procalcitonin in serum during the infection, (20) Maximal white cells count (WBC) during the infection [*leucopenia, as a potential marker of SIRS was also recorded (if it had been reported)], (21) Presence of clinical signs of systemic infection (related to body temperature, heart rate, blood pressure and respiratory rate), (22) Additional morphological, radiography and/or endoscopic diagnostic methods which confirmed the invasiveness of infection (findings of radiographic imaging procedures such as X-ray or ultrasound scans or computerized tomography scans or magnetic resonance imaging examinations), (23) Altered specific hematological and biochemical laboratory parameters that suggested infections of organs/tissues, (24) Confirmed postoperative infection, (25) Antibiotic regimen used, (26) Cure rate and mortality after antibiotic treatment, (27) Adverse events rate, and (28) In vitro susceptibility of $E$. ramosum to antibiotics. The data were extracted by three investigators independently (MM, MK, and RZZ) and then the comparison of the three tables was made by another two investigators (SS and SJ), who produced the final extraction table.

Four researchers (SS, MM, JM and MS) assessed the risk of bias independently for each selected study separately (in accordance with their methodological approaches), while analyzing the following relevant potential sources: attrition bias, selection bias, information bias, and outcome (selective) reporting bias. The final assessment was provided by a senior investigator (SJ). The outcome reporting bias was assessed by determining the proportion of reported target results. In addition, the included studies were arranged according to their geographical distribution, and then the uniformity of the tabulation was estimated.

Then, relevant outcomes were measured in this systematic review: total study duration, age of patients, total number of patients, and maximal serum level of CRP, maximal serum level of procalcitonin and maximal WBC count during the infection as continuous outcomes, while the gender of patients, presence of comorbidities, methods of E. ramosum identification, morphological diagnosis confirming the invasiveness of infection, altered specific hematological and biochemical laboratory parameters that suggested infections of organs/tissues, confirmed postoperative infection, type of invasive infections caused by E. ramosum, type and dose regimen of antibiotics used to treat $E$. ramosum infection, outcomes of antibiotic treatment (cure rate and mortality), adverse events rate, and sensitivity/ resistance rate of E. ramosum to antibiotics, were chosen as categorical outcomes. Individual participants from the included studies were identified to be a unit of analysis. Prior to this analysis, outcomes based on observation data were ascertained only once.

The problem of addressing the missing data included the following: (i) the authors of the original paper were 
directly requested to provide it; (ii) a reference to the results of retrieved papers if disclosed on ClinicalTrial. gov; and (iii) the Discussion section commented on the impact of the missing data on the final results of this review. The assessment of the presence of heterogeneity was not applicable to this type of systematic review.

Standard methods of descriptive statistics were used to describe and summarize the results on a small sample: median and interquartile range (IQR) for continuous variables; number and percentage for categorical variables.

\section{RESULTS}

Literature search results are summarized in Figure 1. The final analysis covered a total of 15 studies that met the inclusion criteria. Among them, there were 11 case reports and 4 case series that reported a total of 19 individual cases. The median age of the subjects with reported invasive infection caused by E. ramosum was 66 (IQR $26,74)$, with the oldest being 91 years old, while the two youngest were 3 years and 6 months old, respectively. A little more than half of the cases were males (11/19 or $57.9 \%$ ). Various types of cancers were the most commonly reported comorbidities/immunocompromising conditions $(4 / 19$ or $21 \%)$, followed by chronic renal failure treated under dialysis (2/19 or $10.5 \%)$, diabetes mellitus (2/19 or $10.5 \%)$ and infected deep-pressure ulcers $(2 / 19$ or $10.5 \%)$; other morbid conditions were reported sporadically (Table 1). Two children (10.5\% of the total number of cases) with otitis media were identified as being predisposed to E. ramosum invasive infections, of whom the older child (5 years old) presented with a chronic suppurative form, a cerebellar abscess that was an intracranial complication (Table 1). E. ramosum as the causative etiological agent in postoperative infections was reported in three patients (15.8\%) (Table 1). Figure 2 depicts the geographical distribution of cases involved in this systematic review. Most reported cases were from the USA (6/19 or $31.6 \%$ ), followed by Spain (3/19 or $15.8 \%)$, Switzerland (2/19 or $10.5 \%)$, France ( $2 / 19$ or $0.5 \%$ ) and Korea (2/19 or $10.5 \%)$. Japan, India, Saudi Arabia and Turkey reported only one case each $(5.3 \%)$. All patients involved were treated exclusively in hospitals. A case series lasting 17 years $^{4}$ was the longest study included in the analysis. In addition to an overview of individual cases, Table 1 also shows an assessment of the risk of bias for each included study.

Blood culture was the most commonly used test used to detect E. ramosum (10/19 or 52.6\%) (Table 1). E. ramosum as the cause of infection was most often initially identified exclusively by biochemical methods (7/19 or 36.8\%), while

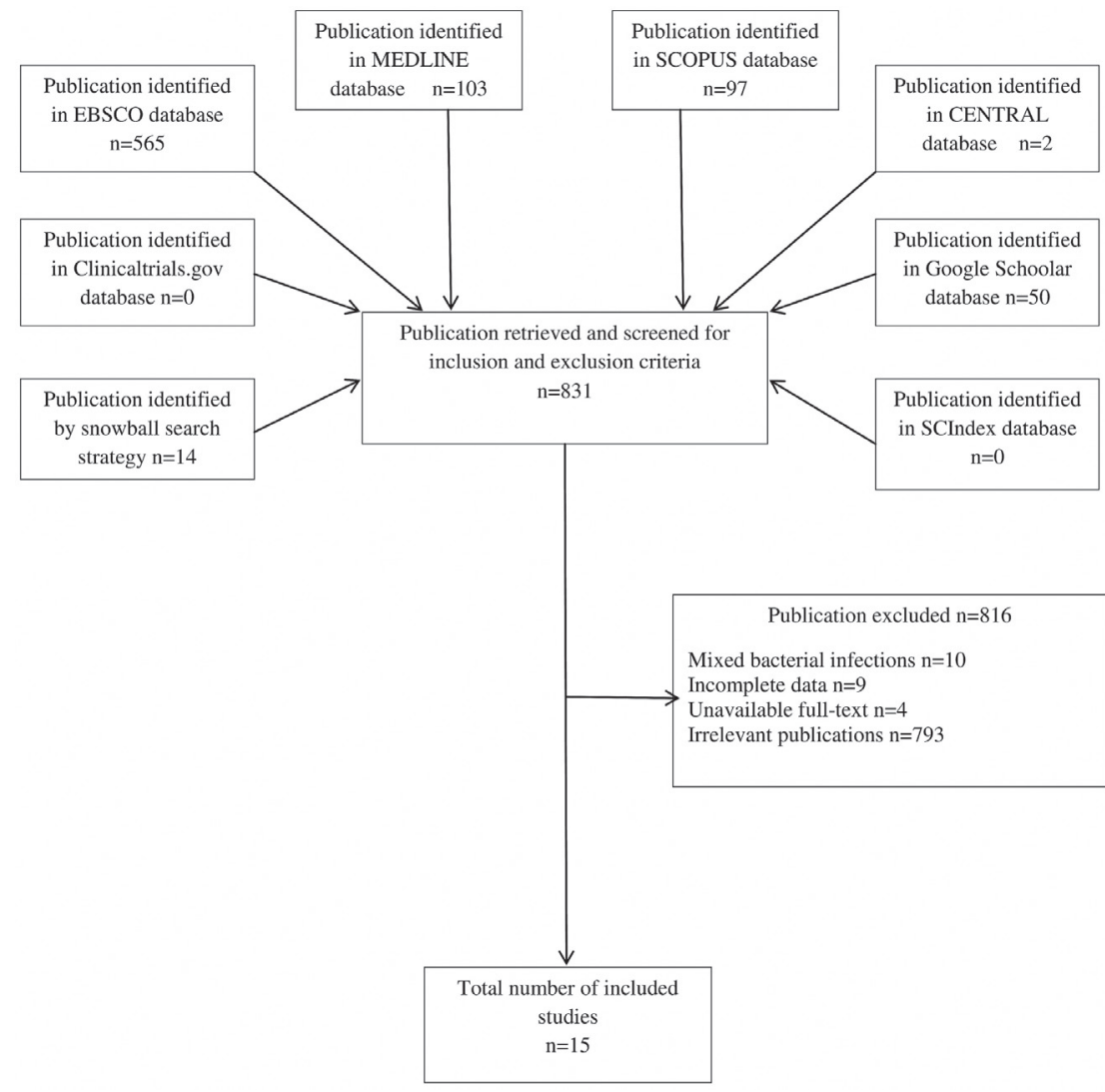

Figure 1 - Selection of studies. 


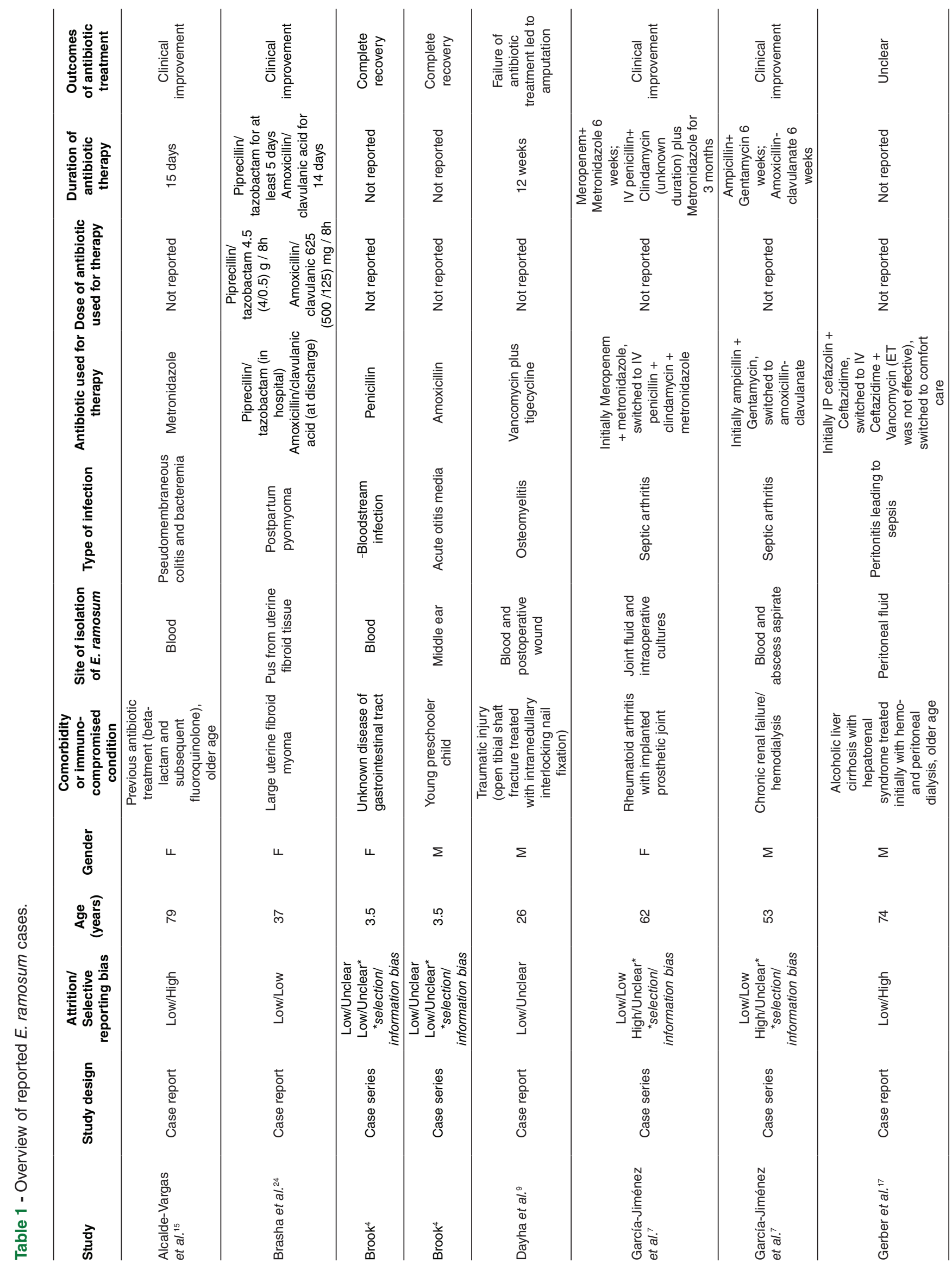




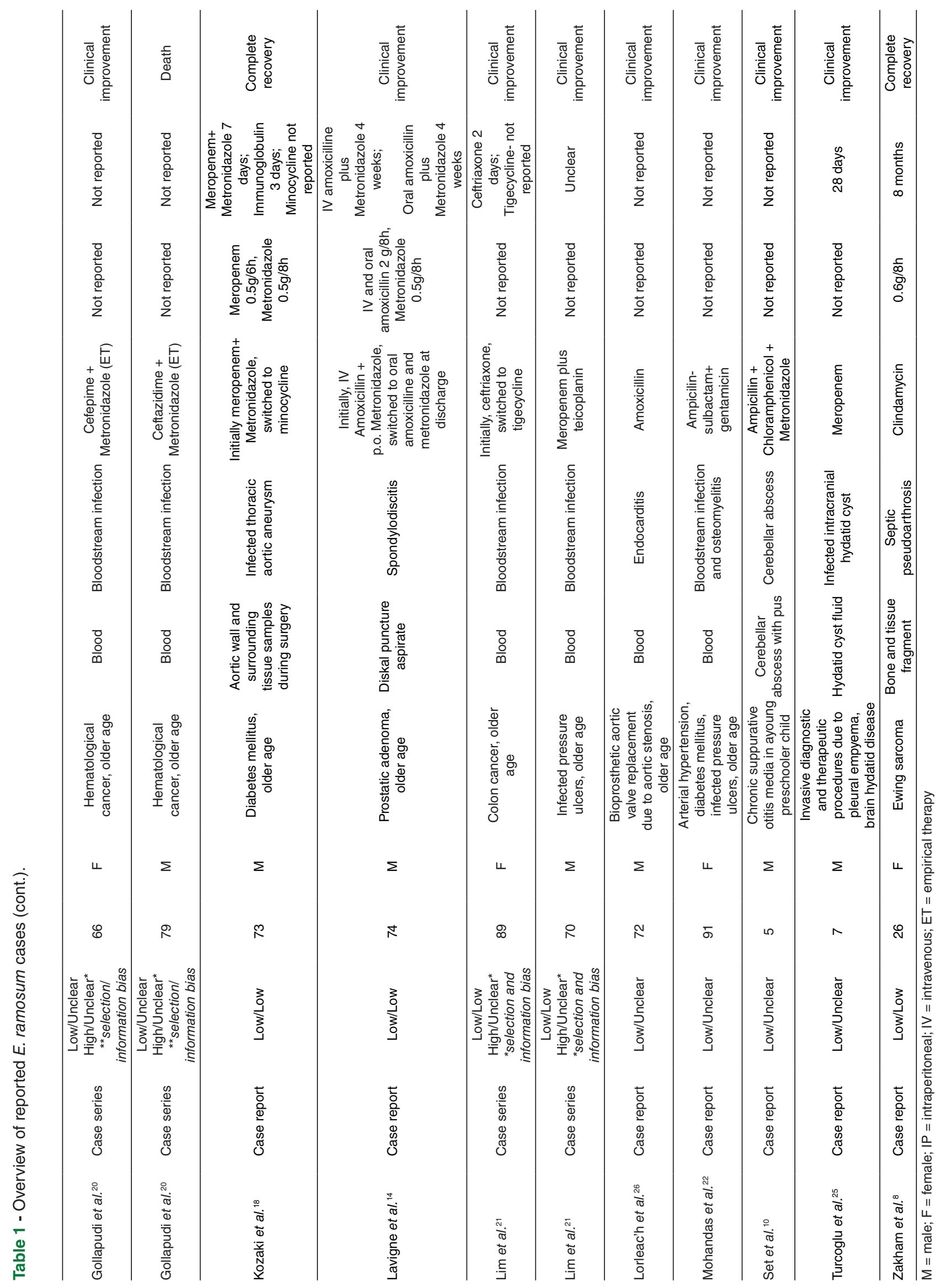




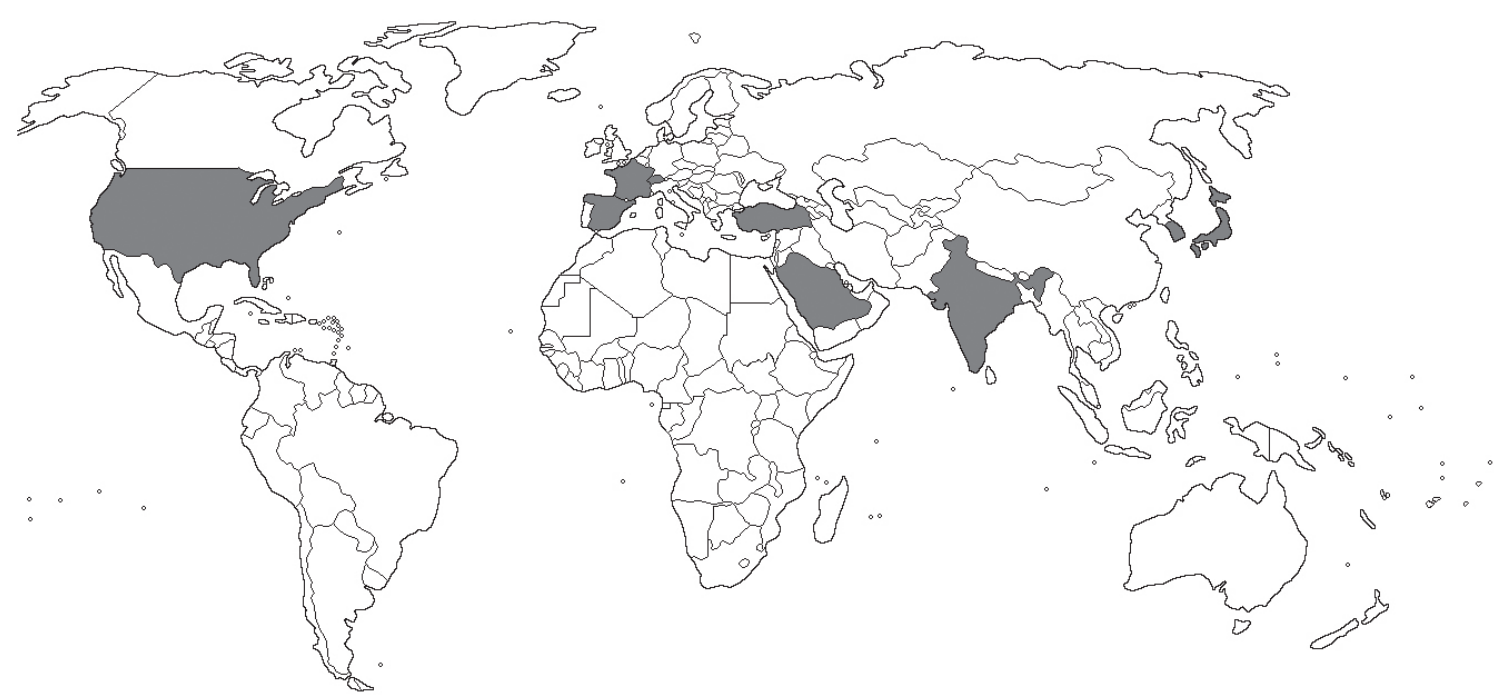

Figure 2 - Geographical distribution of Erysipelatoclostridium ramosum studies. Countries in which E. ramosum was isolated are shaded in gray. Source: Wikipedia Commons ${ }^{30}$.

additional confirmation using $16 \mathrm{~S}$ rRNA gene sequencing was carried out in three cases (15.8\%) cases. Combination of these two methods with MALDI-TOF mass spectrometry (i.E. a triple diagnostic method) was used in three (15.8\%) cases. The 16S rRNA sequencing, as the only method for the identification of $E$. ramosum, was performed in two patients $(10.5 \%)$, as was the use of gas-liquid chromatography alone $(2 / 19$ or $10.5 \%)$. Finally, in two cases $(10.5 \%)$, the method of bacterial identification was not specified.

Thirteen patients (68.4\%) had signs and symptoms of systemic infection, with fever being the most common symptom ( $12 / 19$ or $63.2 \%)$. None of the potential indicators of systemic inflammatory response to infection, such as fever, tachycardia, tachypnea and hypotension, were reported in six patients (31.6\%) included in our study. The level of CRP during infection was measured in only four cases, with the following maximal findings: 51, 166, 278.31 and $31.4 \mathrm{mg} / \mathrm{L}$ (median 108.5, IQR 36.3, 250.2). In addition, in five cases, the maximum WBC count at any time during infection was reported, with the following measured values: $11.1 \times 10^{9} / \mathrm{L}, 11.6 \times 10^{9} / \mathrm{L}, 12.0 \times 10^{9} / \mathrm{L}, 12.5 \times 10^{9} / \mathrm{L}$ and $16.9 \times 10^{9} / \mathrm{L}\left(\right.$ median $12.0 \times 10^{9} / \mathrm{L}$, IQR $11.4 \times 10^{9} / \mathrm{L}$, $14.7 \times 10^{9} / \mathrm{L}$ ). The maximum level of procalcitonin was not reported in any of the patients included in our study. The additional morphological, radiographic and/or endoscopic diagnostic procedures were performed in more than a half of the cases (11/19 or 57.9\%), and the following diagnoses were confirmed: septic arthritis and osteomyelitis in two cases each $(10.5 \%)$, while pseudomembranous colitis, spondylodiscitis, pyomyoma, endocarditis, cerebellar abscess, infection of intracranial hydatid cyst and infection of thoracic aortic aneurysm in one case each $(5.3 \%)$. Altered laboratory findings indicating a specific organ/ tissue infection were reported in two cases: a patient under peritoneal dialysis and presenting with peritonitis with more than $7,000 / \mathrm{mm}^{3} \mathrm{WBC}$ (with predominance of neutrophils) measured in the dialysate, and in patient with septic arthritis, $95,000 / \mathrm{mm}^{3} \mathrm{WBC}$ ( $94 \%$ of neutrophils) measured in the joint fluid. Altogether, E. ramosum was most often found to be a causative agent of bloodstream infections (6/19 or $31.6 \%$ ) (Table 1).

Antimicrobial susceptibility testing of E. ramosum was performed in eight patients (42.1\%), and the summarized susceptibility pattern of these isolates is depicted in Figure 3. Almost two thirds of all isolates ( $5 / 8$ or $62.5 \%$ ), or isolates collected from five of the total number of 19 included cases, were tested for susceptibility to metronidazole and penicillin, and the sensitivity of E. ramosum to metronidazole was confirmed in all five cases (susceptibility of $100 \%$ ), while in one patient this bacterium was resistant to penicillin (susceptibility of $80 \%$ ). In the studies in which more than two isolates were reported to have being tested for the susceptibility to specific antibiotics, they were all susceptible to amoxicillin/clavulanate (4/12 or 33.3\%) and piperacillin/tazobactam (3/12 or $25 \%)$. Finally, in addition to penicillin, individual cases of E. ramosum resistant to clindamycin, ciprofloxacin, imipenem and ertapenem were also reported.

Treatment of E. ramosum invasive infections in a majority of the patients (12/19 or $63.2 \%$ ) comprised more than one antimicrobial drug, while mono antibiotic therapy was used in seven cases (36.8\%). Metronidazole was the most commonly used antibiotic (7/19 or 36.8\%), followed by meropenem (5/19 or $26.3 \%)$, and amoxicillin (4/19 or $21 \%$ ) (Figure 4$)$. In most patients (12/19 or $63.2 \%$ ) a relevant clinical improvement was reported after antibiotic 


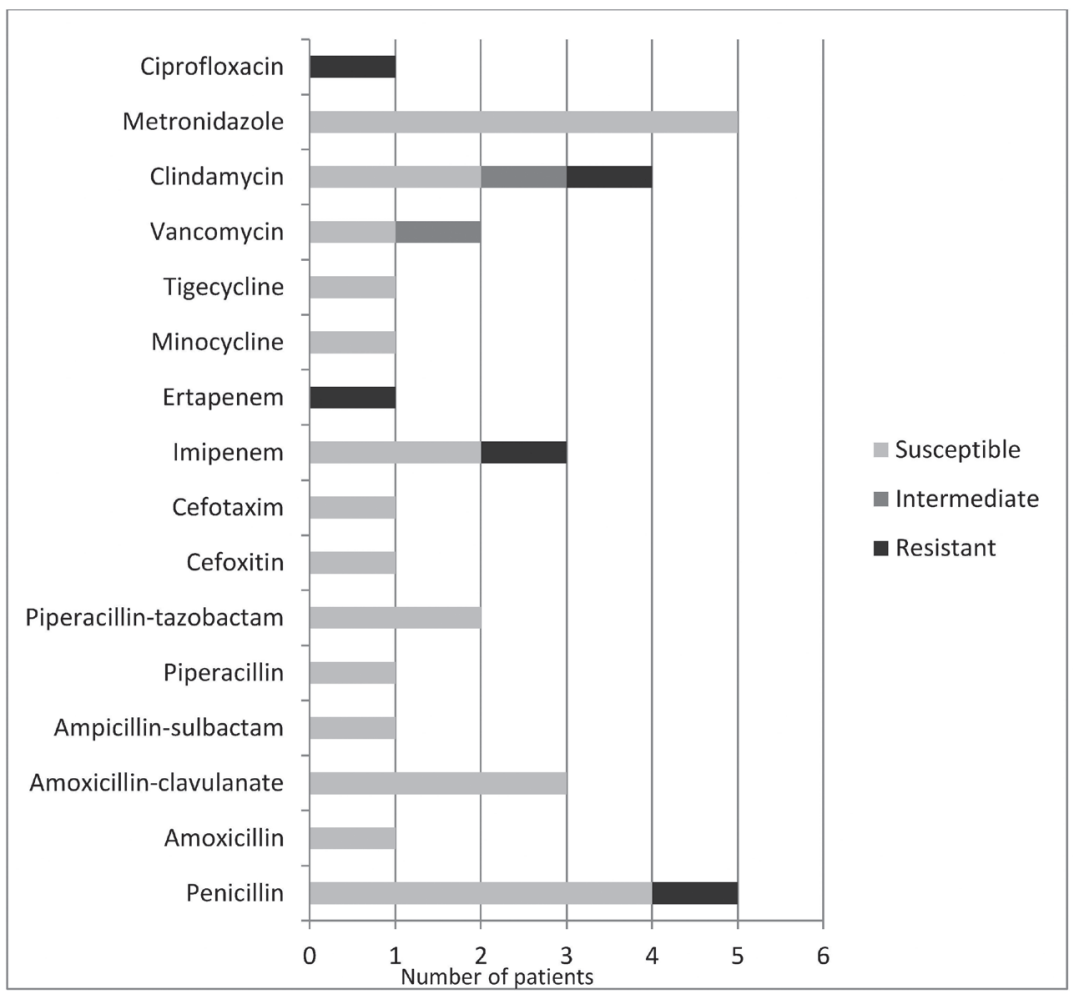

Figure 3 - Susceptibility of E. ramosum to antibiotics.

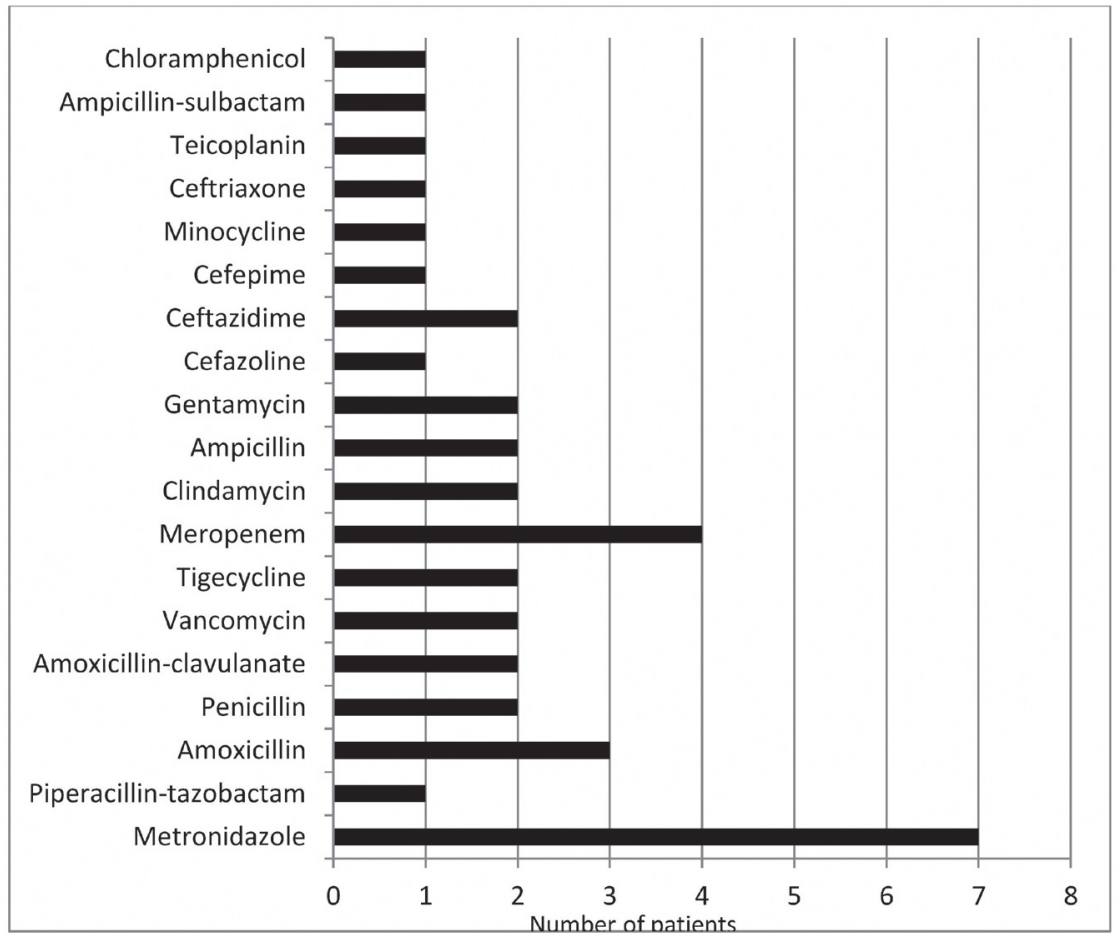

Figure 4 - Antibiotics used for treatment of $E$. ramosum infections.

therapy, while four patients (21\%) experienced a complete recovery. A fatal outcome was reported in one patient with bacteremia, and in one patient with osteomyelitis that underwent amputation due to the antibiotictherapy
failurE. Finally, the antibiotic therapy effectiveness was not completely clear in one patient with peritonitis. Adverse effects of the applied antibiotics were reported only for clindamycin, which was discontinued in a patient with 
pseudoarthritis after eight months of continuous therapy due to the occurrence of serious gastrointestinal side effects. The antibiotic treatment used in individual patients is shown in Table 1.

\section{DISCUSSION}

This systematic review confirmed the clinical relevance of $E$. ramosum as a cause of various severe infections mainly in immunocompromised inpatients. These opportunistic infections, of which bloodstream infections were the most commonly reported, affected both, children and adults, with a predilection for elderly patients. The most common chronic underlying diseases associated with immunodeficiency were different types of cancer, diabetes mellitus and end-stage renal disease treated under hemodialysis and/or peritoneal dialysis, similarly to the emergence of infections by other anaerobic pathogens ${ }^{6}$. E. ramosum was most often isolated from blood culture and identified by specific biochemical tests, in a little more than $50 \%$ of the total number of patients and almost $40 \%$ of all cases, respectively. In one-sixth of all cases, novel sophisticated molecular techniques, such as MALDI-TOF mass spectrometry and 16S rRNA gene sequencing, were used to confirm the identification of isolated E. ramosum. This bacterial species was also recovered from different infection sites, depending on the infected tissuE. Serious infections caused by E. ramosum were in most cases effectively treated with antibiotics, except in two patients, one of whom died. More than one isolate of E. ramosum exhibited $100 \%$ of susceptibility to metronidazole, amoxicillin/clavulanate and piperacillin/ tazobactam, constrasting with individual strains of this bacterium resistant to penicillin, ciprofloxacin, clindamycin, imipenem and ertapenem that were also reported. Along with metronidazole, meropenem was the most commonly used highly effective antibiotic, which was given as part of initial empirical antibiotic therapy.

Being a normal resident of the human intestinal flora, E. ramosum plays only in rare occasions a pathogenic role, leading to invasive infections most commonly in older adults with any type or degree of compromised immune system. Our review included reports of various of these infections that could be reliably associated with immunosuppressive conditions, such as: (i) septicemia in patients with hematological cancers ${ }^{20}$ or colon carcinoma ${ }^{21}$ or in a frail elderly, a bedridden patient presenting with multiple severely infected pressure ulcers ${ }^{21}$; (ii) septicemia and osteomyelitis ${ }^{22}$ or infected thoracic aortic aneurysm ${ }^{18}$ in patients with diabetes mellitus; (iii) septic arthritis in a patient with rheumatoid arthritis after prosthetic joint replacement or in a case of chronic renal failure under hemodialysis ${ }^{7}$; (iv) peritonitis followed by sepsis in patient with hepatorenal syndrome under hemo- and peritoneal dialysis ${ }^{17}$ and septic pseudoarthrosis in a case of Ewing's sarcoma ${ }^{8}$. Interestingly, similar to the best known and more important human opportunistic pathogen from the Clostridia class, i.E. Clostridium difficile ${ }^{23}$, E. ramosum was also found to be a causative agent of pseudomembranous colitis and bacteremia, in an advanced aged patient after a previous use of wide spectrum antibiotics (fluoroquinolones and beta-lactams $)^{15}$. In addition, E. ramosum caused an acute middle ear infection ${ }^{4}$ leading to an intracranial abscess as a complication of a suppurative chronic otitis media $^{10}$ in a young child. As mentioned earlier, these two target populations of patients have long been recognized in terms of susceptibility to E. ramosum infections ${ }^{4,5}$. A high risk for E. ramosum infection is likely to be associated with underdevelopment and dysfunction of the intestinal mucosal barrier in young children and immunodeficient adults/elderly patients, that enable the unique virulence factors (IgA proteases) of this commensal anaerobic bacterium to help invading the host and cause infection at various locations, scaping the host's defense ${ }^{5}$. However, rare reports described E. ramosum as the causal agent of invasive infections in apparently immunocompetent individuals with complicated benign disorders or with indwelling medical devices due to sepsis after bowel perforation, unknown benign gastrointestinal disease in a preschool child ${ }^{4}$, postpartum pyomyoma ${ }^{24}$, spondylodiscitis in an older patient with benign prostatic hyperplasia ${ }^{14}$, secondary infection in a hydatid cyst located in the brain of a 7-year old child previously subjected to an invasive diagnosis and therapeutic procedures due to pleural empyema ${ }^{25}$, endocarditis in an elderly patient with previous bioprosthetic aortic valve replacement ${ }^{26}$, and osteomyelitis after traumatic injury (open long bone fracture) ${ }^{9}$. The latter calls attention to the potential ubiquitous nature of the E. ramosum spores, which, like other Clostridium species, have probably originated from the soil and entered the body through an open traumatic wound ${ }^{9}$.

E. ramosum, together with Clostridium innoculum and Clostridium clostridioforme, belongs to a unique RIC group of clostridia (abbreviation is derived from the initial letters of the names of the bacteria; R- ramosum, I- innoculum, C-clostridioforme), that are often misidentified or are not observed in clinical isolates by routine microbiological methods due to difficulties in distinguishing them from other anaerobic bacteria. The reason for is the inconsistent Gram staining pattern (usually negative at first), the typical formation of terminal spores that may be absent or difficult to identify, as well as atypical colony morphology $y^{6,22,27}$. These factors probably explain the belief that infections 
caused by E. ramosum, based on its correct identification as the only pathogen present in positive cultures, are underestimated in routine clinical practicE. According to the ability of E. ramosum to produce acetic acid and ferment certain mono- and disaccharides as well as sugar alcohols ${ }^{5}$, in most cases covered by our review, this bacterium was identified by using conventional biochemical techniques, such as API test systems (BioMérieux SA, Marcy-l'Étoile, France). Although these phenotype-based tests are not entirely perfect in identifying $E$. ramosum in relation to other anaerobes ${ }^{27}$, this review generally indicates their good reliability. However, the non-selectivity of this identification kit was reported to be related to different biochemical reactions used and their inability to differentiate E. ramosum from Actinomyces israelii with certainty; in this case, $16 \mathrm{~S}$ rRNA gene sequencing was used as the gold standard for confirming that it was a $E$. ramosum isolate $^{14}$. MALDI-TOF mass spectrometry was also used in some cases $^{8,21}$ as a revolutionary, more attractive and accurate method for identifying bacteria based on their protein profile, but definitive confirmation of $E$. ramosum appears to require further use of bacterial genome analyzes ${ }^{8}$. As there is no routine laboratory identification of this bacterium, current state-of-the-art method, such as 16S rRNA gene sequencing, should be used whenever possible, either in combination or as the only diagnostic method to confirm E. ramosum isolates, especially in culture-negative infections.

As mentioned earlier, although antibiotics exhibiting bactericidal activity to E. ramosum are generally effective, either in monotherapy or in combination regimen, the recommended treatment for infections caused by this specific clostridium species has not yet been defined. Our review confirmed good in vitro susceptibility of E. ramosum mainly to metronidazole and two broadspectrum beta-lactams plus beta-lactamase inhibitors (i.E. amoxicillin/clavulanate and piperacillin/tazobactam, respectively). In addition, another broad-spectrum betalactam (carbapenem) antibiotic, meropenem, showed high efficacy after frequent use, mostly without prior in vitro activity testing. This is not surprising since these drugs have long been recognized as the antimicrobial agents of choice or alternative antibiotics for the treatment of various anaerobic infections, including those caused by clostridial species $^{28}$. Favorable pharmacokinetic characteristics, especially wide distribution in almost all tissues, probably contribute to their efficacy in the treatment of the reported E. ramosum infections in different sites $7,10,14,15,18,20,21,24,25$. Other potentially effective treatment options, depending on the type and severity of the infection, include ampicillin/ sulbactam, imipenem, glycopeptides (vancomycin or teicoplanin) and chloramphenicol.
Previous studies showed that E. ramosum strains producing beta-lactamases exhibited resistance to penicillin in $20 \%$ of cases $^{27-29}$, so this antibiotic could not be recommended for the treatment of infections caused by $E$. ramosum due to the potential therapeutic failurE. Similarly, the use of clindamycin or cephalosporins should also be avoided, because $E$. ramosum often shows variable susceptibility to these antibiotics, as other clostridial species $\mathrm{do}^{27,28}$. Besides, the use of clindamycin in the treatment of severe invasive infections should not be practiced due to possible serious gastrointestinal side effects $^{8}$. As mentioned previously, the antibiotic resistance in E. ramosum strains is not widespread. This anaerobic bacillus showed a high level resistance only to rifampin, aminoglycosides, fluoroquinolones and tetracyclines ${ }^{5,14}$. However, certain tetracyclines, especially broad-spectrum minocycline ${ }^{18}$ or tigecycline ${ }^{21}$, might be possible treatment options for E. ramosum infections, when its in vitro susceptibility has been previously documented. Failure of antibiotic therapy despite the use of multiple antibiotics with proven in vitro activity on E. ramosum that can result in death or other serious complications is likely to be associated with uncontrolled progression of a severe underlying disease or with massive bacterial invasion associated with an extremely weak immune response of the host, as occurred in patients covered by this review ${ }^{9,17,20}$. In addition, E. ramosum is often being isolated as a component of polymicrobial-mediated infections $\mathrm{s}^{4,5,11-13}$ that are particularly difficult to treat and have unpredictable clinical outcomes.

This systematic review has the following shortcomings: (i) a relatively small number of reported cases with invasive infections caused by $E$. ramosum that was analyzed; (ii) $16 \mathrm{~S}$ rRNA gene sequencing as the most accurate method for confirming E. ramosum in different isolates was used in only a few patients, implying a controversial reliability in terms of correct identification of this anaerobic bacterium; and (iii) in several analyzed reports, relevant information on the susceptibility of E. ramosum to antibiotics, as well as the description of all outcomes of the treatment of infections, were either missing or incomplete

In conclusion, E. ramosum rarely plays a pathogenic role in causing serious invasive infections of various tissues in predisposed patients of any age, predominantly in those with significant level of immunosuppression. The 16S rRNA gene sequencing should be used independently of other phenotype-based methods and whenever possible, to accurately identify this bacterium. Metronidazole, a broad-spectrum beta-lactam with a beta-lactamase inhibitor and meropenem appear to be the antibiotics of choice that should be used in combination or as a monotherapy to treat 
E. ramosum infections. Other antimicrobial agents, such as imipenem, glycopeptide antibiotics, chloramphenicol and tigecycline should only be used based on their in vitro activity findings.

\section{ACKNOWLEDGMENTS}

This study was partially financed by grant $\mathrm{N}^{\mathrm{o}} 175007$, from the Serbian Ministry of Education, Science and Technological Development.

\section{AUTHORS' CONTRIBUTIONS}

MM designed the study; MM, SS, JM, MK, RZZ and MS performed the literature search and data analysis; SJ critically reviewed the work.

\section{CONFLICT OF INTERESTS}

The authors declare no conflict of interests.

\section{REFERENCES}

1. Parks DH, Chuvochina M, Waite DW, Skarhewski A, Chaumeil PA, Hugenholtz P. A standardized bacterial taxonomy based on genome phylogeny substantially revises the tree of lifE. Nat Biotechnol. 2018;36:996-1004.

2. Tally FP, Armfield AY, Dowell VR Jr, Kwok YY, Sutter VL, Finegold SM. Susceptibility of Clostridium ramosum to antimicrobial agents. Antimicrob Agents Chemother. 1974;5:589-93.

3. Kosowska K, Reinholdt J, Rasmussen LK, Sabat A, Potempa J, Kilian M, et al. The Clostridium ramosum IgA proteinase represents a novel type of metalloendopeptidasE. J Biol Chem. 2002;277:11987-94

4. Brook I. Clostridial infection in children. J Med Microbiol. 1995;42:78-82.

5. Forrester JD, Spain DA. Clostridium ramosum bacteremia: case report and literature review. Surg Infect (Larchmt). 2014;15:343-6

6. Gajdács M, Urbán E. The relevance of anaerobic bacteria in brain abscesses: a ten-year retrospective analysis (2008-2017). Infect Dis (Lond). 2019;51:779-81.

7. García-Jiménez A, Prim N, Crusi X, Benito N. Septic arthritis due to Clostridium ramosum. Semin Arthritis Rheum. 2016;45:617-20

8. Zakham F, Pillonel T, Brunel AS, Zambelli PY, Greub G, Croxatto A, et al. Molecular diagnosis and enrichment culture identified a septic pseudoarthrosis due to an infection with Erysipelatoclostridium ramosum. Int J Infect Dis. 2019;81:167-9.
9. Dahya V, Ramgopal M, Collin B, Robinson M. Clostridium ramosum Osteomyelitis in an immunocompetent patient after traumatic injury. Infect Dis Clin Pract. 2015;23:102-4.

10. Set R, Kandian S, Koppikar GV. Clostridium ramosum in a case of cerebellar abscess. Indian J Med Microbiol. 2001;19:149-50.

11. Nanda N, Voskuhl GW. Lung abscess caused by Clostridium ramosum. J Okla State Med Assoc. 2006;99:158-60

12. Takano N, Yatabe MS, Yatabe J, Kato M, Sueoka D, Iguchi $\mathrm{S}$, et al. Fatal Fournier's gangrene caused by Clostridium ramosum in a patient with central diabetes insipidus and insulin-dependent diabetes mellitus: a case report. BMC Infect Dis. 2018;18:363.

13. van der Vorm ER, von Rosenstiel IA, Spanjaard L, Dankert J. Gas gangrene in an immunocompromised girl due to a Clostridium ramosum infection. Clin Infect Dis. 1999;28:923-4.

14. Lavigne JP, Bouziges N, Sotto A, Leroux JL, Michaux-Charachon S. Spondylodiscitis due to Clostridium ramosum infection in an immunocompetent elderly patient. J Clin Microbiol. 2003;41:2223-6.

15. Alcalde-Vargas A, Trigo-Salado C, Leo Carnerero E, De-la-CruzRamírez D, Herrera-Justiniano JM. Pseudomembranous colitis and bacteremia in an immune competent patient associated with a rare specie of Clostridium (C. ramosum). Rev Esp Enferm Dig. 2012;104:498-9.

16. Muakkassa WF, Mohanty PK, Kipreous B, Lee HM, Goldman MH. Left ventricular mass with septic (Clostridium ramosum) arterial emboli in a renal allograft patient: report of a case and review of the literaturE. Transplant Proc. 1983;15:1715-9.

17. Gerber JS, Berney-Meyer L, Segerer S. Clostridium Ramosum: a rare cause of peritoneal dialysis-related peritonitis. Perit Dial Int. 2018;38:231-2.

18. Kozaki S, Miyamoto S, Uchida K, Shuto T, Tanaka H, Wada T, et al. Infected thoracic aortic aneurysm caused by Clostridium ramosum: a case report. J Cardiol Cases. 2019;20:103-5.

19. Gajdács M, Spengler G, Urbán E. Identification and antimicrobial susceptibility testing of anaerobic bacteria: Rubik's cube of clinical microbiology? Antibiotics (Basel). 2017;6:25.

20. Gollapudi LA, Narurkar R, Wang G, Dhand A. Clostridium ramosum (C. ramosum) bacteremia: single-center study. Open Forum Infect Dis. 2017;4 Suppl 1:S556.

21. Lim YK, Oh SM, Kweon OJ, Lee M. Two cases of bacteremias caused by Clostridium ramosum. Ann Clin Microbiol. 2015;18:98-101.

22. Mohandas R, Poduval RD, Unnikrishanan D. Clostridium ramosum bacteremia and osteomyelitis in a patient with infected pressure sores. Infect Dis Clin Pract. 2001;10: 123-4.

23. Brown KA, Khanafer N, Daneman N, Fisman DN. Metaanalysis of antibiotics and the risk of community-associated Clostridium difficile infection. Antimicrob Agents Chemother. 2013;57:2326-32. 
24. Brasha N, Abbas M, Gadeer A, Gadeer R, Ali A. Postpartum pyomyoma caused by Clostridium Ramosum: a case report Clin Experim Obstetr Gynecol. 2018;45:636-40.

25. Turkoglu OF, Solaroglu I, Tun K, Beskonakli E, Taskin Y. Secondary infection of intracranial hydatid cyst with Clostridium ramosum. Childs Nerv Syst. 2005;21:1004-7.

26. Lorleac'h A, Cazanave C, Pereyre S, Kuli B, Neau D, Ragnaud JM. Une endocardite à Clostridium ramosum: premier cas décrit? Med Malad Infect. 2008;38:S146.

27. Alexander CJ, Citron DM, Brazier JS, Goldstein EJ. Identification and antimicrobial resistance patterns of clinical isolates of Clostridium clostridioforme, Clostridium innocuum, and
Clostridium ramosum compared with those of clinical isolates of Clostridium perfringens. J Clin Microbiol. 1995;33:3209-15.

28. Brook I. Spectrum and treatment of anaerobic infections. J Infect Chemother. 2016;22:1-13.

29. Leal J, Gregson DB, Ross T, Church DL, Laupland KB. Epidemiology of Clostridium species bacteremia in Calgary, Canada, 2000-2006. J Infect. 2008;57:198-203.

30. Wikimedia Commons. Blank map political world territories. png. [cited 2021 Mar 3]. Available from: https://commons. wikimedia.org/wiki/File:Blank_map_political_world_ territories.png 